\title{
Neurocysticercosis: Clinical Characteristics and Changes from 26 Years of Experience in an University Hospital in Korea
}

\author{
Hyo-Ju Son ${ }^{1}$, Min Jae Kim ${ }^{1, *}$, Kyung Hwa Jung1', Sungim Choi', Jiwon Jung', Yong Pil Chong1', Sung-Han Kim', \\ Sang-Oh Lee', Sang-Ho Choi', Yang Soo Kim', Jun Hee Woo', Bong-Kwang Jung ${ }^{2}$, Hyemi Song ${ }^{2}$, Jong-Yil Chai ${ }^{2}$

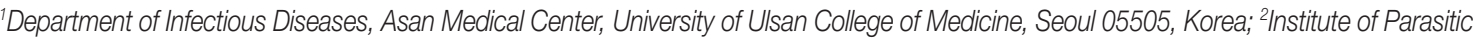 \\ Diseases, Korea Association of Health Promotion, Seoul 07649, Korea
}

\begin{abstract}
The prevalence of human taeniasis has decreased in Korea. The stool egg positive proportion decreased from $1.9 \%$ in 1971 to $0 \%$ in 2004 in nationwide surveys. The neurocysticercosis (NCC) is also presumed to decrease. However, detailed information regarding the recent status of NCC in Korea is lacking. We retrospectively reviewed NCC cases from 1990 to 2016 at Asan Medical Center, a 2700-bed tertiary referral hospital in Korea. We identified patients based on clinical symptoms, brain imaging, pathology and serological assay. The cases were classified as parenchymal, extraparenchymal, and mixed NCC. Eighty-one patients were included in the analysis. The mean age was 54.5 years, and $79.0 \%$ were male. The number of NCC cases was highest from 1995 to 1999, and continuously decreased thereafter. Forty (49.4\%) patients had parenchymal NCC, while 25 (30.9\%) patients had extraparenchymal NCC, and 16 (19.8\%) patients had mixed NCC. The seizure and headache were most common symptom of parenchymal NCC and extraparenchymal NCC respectively. Hydrocephalus was more common in extraparenchymal NCC, and patients with extraparenchymal NCC were more likely to require a ventriculoperitoneal shunt. Cases of NCC are decreasing accordingly with human taeniasis and lesion location was the most important determinant of clinical presentation and outcome of NCC in Korea.
\end{abstract}

Key words: Taenia solium, neurocysticercosis, human taeniasis, Korea

\section{INTRODUCTION}

Neurocysticercosis (NCC) is an infection of the central nervous system caused by the larval stage of the pork tapeworm Taenia solium [1]. Human NCC is acquired through ingestion of infective T. solium eggs. Multiple body tissues such as the eyes, skin and muscles can be involved, but the larvae display a strong affinity for the central nervous system [2]. Currently, NCC is endemic in Latin America, Africa and Asian countries, i.e., India, China, and Nepal [3].

The prevalence of human taeniasis has recently decreased in Korea. According to a nationwide survey, human taeniasis was common in Korea until the 1980s. The stool egg positive proportion was $1.9 \%$ in 1971 , decreased to $0 \%$ in 2004 , and remained less than $0.1 \%$ thereafter [4]. The introduction of an-

\footnotetext{
- Received 17 March 2019, revised 9 May 2019, accepted 10 May 2019.

* Corresponding author (nahani99@gmail.com)

(C) 2019, Korean Society for Parasitology and Tropical Medicine

This is an Open Access article distributed under the terms of the Creative Commons Attribution Non-Commercial License (http://creativecommons.org/licenses/by-nc/4.0) which permits unrestricted non-commercial use, distribution, and reproduction in any medium, provided the original work is properly cited.
}

thelminthic medication, changes in pig agriculture, improvements to the sewage system and economic development have likely influenced the decline of human taeniasis. NCC is also presumed to have decreased in Korea, but detailed information regarding recent NCC prevalence is lacking. We thus investigated the clinical features, outcomes and changing trends in NCC in the context of recent declines in human taeniasis.

\section{MATERIALS AND METHODS}

\section{Patients and data collection}

We performed a retrospective review of medical records to identify patients with NCC. We identified patients with a discharge diagnosis of cysticercosis who visited Asan Medical Center, a 2700-bed tertiary referral hospital in Seoul, Korea, from January 1990, to December 2016. Among the patients, those satisfying all of the following conditions were selected: (1) new onset neurological symptoms; (2) computed tomography (CT) or magnetic resonance imaging (MRI) findings suggestive of NCC; and (3) pathologic findings compatible with NCC, or positive cerebrospinal fluid (CSF) ELISA for cys- 
ticercus-specific IgG antibodies. New onset neurological symptoms included headache, seizure, gait abnormality, cranial nerve palsy and focal neurological deficits such as aphasia or hemiparesis. Image findings showing cysts, nodules and calcification in the central nervous system were considered to be suggestive of NCC. Lesion locations were classified as parenchyma of the brain, or inside the ventricles or subarachnoid space. The presence of hydrocephalus and leptomeningeal enhancement were also noted, as were spinal cord lesions.

The patients were initially classified as having parenchymal, extraparenchymal or mixed disease. When the nodules, cysts or calcifications were located only in the parenchyma of brain, the case was classified as parenchymal disease. Extraparenchymal disease included cases with cystic lesions in the ventricles or in the subarachnoid spaces. Cases with both parenchymal and extraparenchymal lesions were classified as mixed disease. According to new guidelines published in 2018 [5], we subdivided parenchymal disease into viable parenchymal and calcified parenchymal disease, and extraparenchymal disease into subarachnoid and intraventricular disease. No patient had a single enhancing lesion in imaging.

For enrolled patients, information regarding demographic characteristics, clinical variables including symptoms and image findings, methods of diagnosis, total follow-up period, laboratory results of CSF, and clinical outcomes was collected. After categorizing patients as parenchymal, extraparenchymal, or mixed, differences in the clinical characteristics and outcomes were compared between each group. A chi-square test or Fisher's exact test was used to compare categorical variables, and a Student $t$-test or Mann-Whitney test was used to compare continuous variables between 2 groups. A linear-by-linear association test was used to analyze trends among multiple groups. Statistical analysis was conducted with SPSS 24.0 software (IBM Corp., Armonk, New York, USA).

\section{Ethical approval}

This study was approved by the Institutional Review Board (IRB) of Asan Medical Center (IRB Number 2017-0346). Informed consent was waived by the IRB considering the retrospective nature of the study.

\section{RESULTS}

A total of 89 patients had a discharge diagnosis of cysticercosis during the study period. Among them, 5 patients had cystic mass lesions in organs other than the central nervous system, such that the diagnosis of cysticercosis was uncertain. Thus, these patients were excluded from analysis. In 3 patients, clinical symptoms and image findings were suggestive of NCC, but both serological test and pathological evidence were absent, so these patients were also excluded from analysis. Finally, 81 patients were included in the analysis.

\section{Epidemiology}

The mean patient age was 54.5 years (standard deviation (SD), 12.7 years) and 64 patients (79.0\%) were male. Male patients were more likely to have parenchymal NCC (54.7\% vs $29.4 \%, P=0.025)$. Forty-eight patients (59.3\%) lived near Seoul where the hospital is located, and 8 patients $(9.9 \%)$ lived in Jeju Island, where cysticercosis was endemic [6]. Patients were followed up for a mean 82.4 months (range, 2-300 months). When patients were grouped according to the year of diagnosis, 33 patients (40.7\%) were diagnosed from 1995 to 1999 . The number of patients diagnosed in each 10-year period continuously decreased thereafter (Fig. 1). The mean age at diagnosis was in the range of 51.6-59.9 years old according to the 10-year period, and the age at diagnosis continued to increase. The mean age at diagnosis was higher in patients diagnosed after 2000 than in patients diagnosed before 1999 (58.8 vs $51.1, P=0.006$ ).

\section{Parenchymal and extraparenchymal NCC}

Among the 81 patients, 58 patients had cysts or nodules in the parenchyme of the brain or spinal cord, and 37 and $7 \mathrm{pa}-$

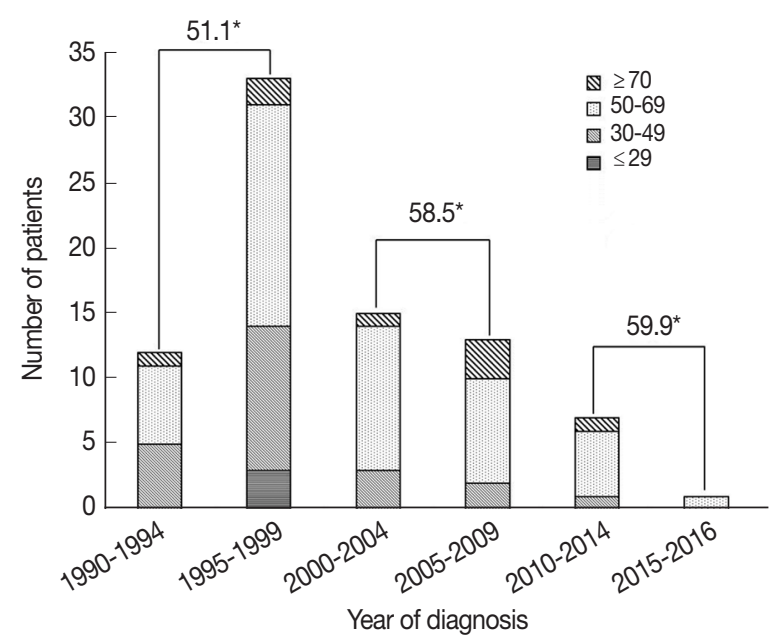

Fig. 1. Numbers of patients, age group distributions and median ages according to period of diagnosis. "Mean age at diagnosis. 
tients had subarachnoid and intraventricular cysts, respectively. Patients were grouped according to lesion location, with 40 patients (49.9\%) classified as parenchymal NCC, 25 patients (30.9\%) classified as extraparenchymal NCC, and 16 patients (19.8\%) classified as mixed NCC (Table 1).

\section{Parenchymal NCC}

The mean patient age of parenchymal NCC was 53.8 years old (SD, 10), and 35 patients (87.5\%) were male. A total of 40 parenchymal NCC patients consisted of 14 viable parenchymal NCC, 8 calcified parenchymal NCC, and 18 patients with both viable and calcified parenchymal NCC lesions. The most common presenting symptom was seizure, followed by headache, focal neurologic deficits, gait disturbances and voiding difficulties, respectively. Hydrocephalus was present in $5 \mathrm{pa}-$ tients (12.5\%), and leptomeningeal enhancement was present in 3 patients (7.5\%). In terms of complications, 2 patients (5.0\%) had uncontrolled seizures and 1 patient $(2.5 \%)$ had uncontrolled increased intracranial pressure.

\section{Extraparenchymal NCC}

The mean age of patients with extraparenchymal NCC was 54.0 years old $(\mathrm{SD}, 16.6)$ and 16 patients $(64.0 \%)$ were male. Twenty $(80.0 \%)$ and $3(12.0 \%)$ patients had subarachnoid

Table 1. Comparison of clinical and radiological characteristics of parenchymal, extraparenchymal and mixed neurocysticercosis

\begin{tabular}{|c|c|c|c|c|c|}
\hline \multirow[t]{2}{*}{ Age at diagnosis, years (mean $\pm S . D)^{c}$} & $\begin{array}{l}\text { Parenchymal } \\
(n=40)(\%)\end{array}$ & $\begin{array}{l}\text { Extra-parenchymal } \\
\qquad(n=25)(\%)\end{array}$ & $\begin{array}{c}\text { Mixed } \\
(n=16)(\%)\end{array}$ & $P 1^{\mathrm{a}}$ & $P 2^{\mathrm{b}}$ \\
\hline & $53.8 \pm 10.8$ & $54.0 \pm 16.6$ & $56.9 \pm 10.7$ & 0.959 & 0.533 \\
\hline \multicolumn{6}{|l|}{ Sex } \\
\hline Male & $35(87.5)$ & $16(64.0)$ & $13(81.3)$ & 0.025 & 0.236 \\
\hline \multicolumn{6}{|l|}{ Symptoms } \\
\hline Headache & $10(25.0)$ & $16(64.0)$ & $8(50.0)$ & 0.002 & 0.375 \\
\hline Seizure & $15(37.5)$ & $0(0)$ & $2(12.5)$ & $<0.001$ & 0.146 \\
\hline Gait disturbance & $4(10.0)$ & $5(20.0)$ & $3(18.8)$ & 0.288 & 0.999 \\
\hline Voiding difficulty & $2(5.0)$ & $0(0)$ & $0(0)$ & 0.519 & N/A \\
\hline Cranial nerve palsy & $4(10.0)$ & $3(12.0)$ & $1(6.2)$ & 0.999 & 0.999 \\
\hline Focal neurological deficit & $7(17.5)$ & $3(12.0)$ & $3(18.8)$ & 0.729 & 0.662 \\
\hline \multicolumn{6}{|l|}{ Involvement of spinal cord } \\
\hline Brain only & $37(92.5)$ & $21(84.0)$ & $14(87.5)$ & 0.282 & 0.757 \\
\hline Spinal cord only & $3(7.5)$ & $4(16.0)$ & $0(0)$ & 0.415 & 0.143 \\
\hline \multicolumn{6}{|l|}{ Image findings } \\
\hline Subarachnoid cyst & $0(0)$ & $22(88.0)$ & $15(93.8)$ & $<0.001$ & 0.545 \\
\hline Intraventricular cyst & $0(0)$ & $5(20.0)$ & $2(12.5)$ & 0.053 & 0.685 \\
\hline Hydrocephalus & $5(12.5)$ & $14(56.0)$ & $4(25.0)$ & $<0.001$ & 0.063 \\
\hline Calcification & $26(65.0)$ & $0(0)$ & $2(12.5)$ & 0.019 & 0.146 \\
\hline Leptomeningeal enhancement & $3(7.5)$ & $5(20.0)$ & $3(18.8)$ & 0.243 & 0.999 \\
\hline \multicolumn{6}{|l|}{ Method of diagnosis } \\
\hline Positive pathology & $14(35.0)$ & $9(36.0)$ & $3(18.8)$ & 0.935 & 0.305 \\
\hline Positive CSF ELISA Ab & $27(67.5)$ & $17(68.0)$ & $13(81.2)$ & 0.967 & 0.350 \\
\hline \multicolumn{6}{|l|}{ CSF characteristics } \\
\hline Cells (/ml) & $37.2 \pm 109.9$ & $46.0 \pm 42.3$ & $69.2 \pm 79.9$ & 0.758 & 0.338 \\
\hline Protein (mg/dl) & $47.8 \pm 20.2$ & $95.6 \pm 50.3$ & $60.5 \pm 24.7$ & 0.002 & 0.078 \\
\hline Glucose (mg/dl) & $65.3 \pm 22.9$ & $35.1 \pm 19.7$ & $61.3 \pm 8.7$ & $<0.001$ & 0.001 \\
\hline \multicolumn{6}{|l|}{ Surgical treatment } \\
\hline Follow-up period in months (mean \pm S.D) & $103.3 \pm 83.2$ & $65.0 \pm 63.6$ & $57.1 \pm 76.3$ & 0.054 & 0.721 \\
\hline Clinical complication & $3(7.5)$ & $6(24.0)$ & $5(31.2)$ & 0.076 & 0.609 \\
\hline Spinal cord compression & $0(0)$ & $2(8.0)$ & $1(6.2)$ & 0.144 & 0.999 \\
\hline Vascular infarction & $0(0)$ & $0(0)$ & $1(6.2)$ & N/A & 0.390 \\
\hline Uncontrolled seizure & $2(5.0)$ & $0(0)$ & $0(0)$ & 0.519 & $\mathrm{~N} / \mathrm{A}$ \\
\hline Uncontrolled ICP & $1(2.5)$ & $4(16.0)$ & $2(12.5)$ & 0.068 & 0.999 \\
\hline Recurrent meningitis & $0(0)$ & $0(0)$ & $1(6.2)$ & $\mathrm{N} / \mathrm{A}$ & 0.390 \\
\hline
\end{tabular}

ap 1: comparing parenchymal and extraparenchymal neurocysticercosis.

${ }^{\mathrm{b}} \mathrm{P} 2$ : comparing extraparenchymal and mixed neurocysticercosis.

SS.D: standard deviation. 
and intraventricular disease, respectively. The remaining 2 patients (8.0\%) had both subarachnoid and intraventricular lesions. In contrast to parenchymal NCC, the most common presenting symptom of extraparenchymal NCC was headache, which presented in 16 patients (64.0\%). No patients presented with seizure. Hydrocephalus was present in 14 patients (56.0\%), and leptomeningeal enhancement was present in 5 patients (20.0\%). Ten patients (40.0\%) required a ventriculoperitoneal (VP) shunt. As a complication, 2 patients (8.0\%) had spinal cord compression and 4 patients (16.0\%) had uncontrolled increased intracranial pressure.

\section{Mixed NCC}

Mixed NCC occurred in 16 patients. Among mixed NCC patients, 15 patients (93.7\%) had subarachnoid cysts and 2 patients (12.5\%) had intraventricular cysts. Calcification was found in 2 patients (12.5\%). Eight patients (50.0\%) presented with headache, and seizure was present in 2 patients $(12.5 \%)$. Four patients (25.0\%) had hydrocephalus, and 3 patients $(18.8 \%)$ had leptomeningeal enhancement in initial radiologic findings.

\section{Comparison of parenchymal and extraparenchymal NCC}

The most common clinical manifestation of parenchymal NCC was seizure (37.5\% parenchymal vs $0 \%$ extraparenchymal, $P<0.001)$. Headache was the most common manifestation of extraparenchymal NCC (25.0\% parenchymal vs $64.0 \%$ extraparenchymal, $P=0.002$ ). Calcification was more common in parenchymal NCC $(65.0 \%$ parenchymal vs $0 \%$ extraparenchymal, $P=0.019)$, and hydrocephalus was more common in extraparenchymal NCC (12.5\% parenchymal vs $56.0 \%$ extraparenchymal, $P<0.001$ ). Patients with extraparenchymal NCC had significantly higher CSF protein $(95.6 \mathrm{mg} / \mathrm{dl}$ vs $47.8 \mathrm{mg} / \mathrm{dl}, P=0.002)$ and lower CSF glucose $(35.1 \mathrm{mg} / \mathrm{dl}$ vs $65.3 \mathrm{mg} / \mathrm{dl}, P<0.001)$, and were more likely to require a VP shunt $(40.0 \%$ vs $2.5 \%, P<0.001)$.

\section{Comparison of extraparenchymal and mixed NCC}

The demographic, clinical, radiological, and inflammatory characteristics of extraparenchymal NCC patients with or without parenchymal cysts were not significantly different. The only difference was lower CSF glucose ( 35.1 vs $61.3, P=0.001$ ) in the extraparenchymal NCC group.

\section{Comparison of viable and calcified parenchymal NCC}

When the patients with viable versus calcified parenchymal NCC were compared, patients with viable parenchymal NCC tended to present with focal neurologic deficits, and those with calcified parenchymal NCC tended to present with seizure. Patients with calcified parenchymal NCC tended to have more clinical complications, including one uncontrolled seizure and one case of uncontrolled intracranial pressure. However, these differences were not statistically significant.

\section{Comparison of subarachnoid and intraventricular extraparenchymal NCC}

Compared with patients with subarachnoid NCC, patients with intraventricular NCC tended to present with headache and to have hydrocephalus. Patients with subarachnoid NCC tended to have more clinical complications, including 2 spinal cord compressions and 4 cases of uncontrolled intracranial pressure. The differences were not statistically significant, likely due to small sample size.

\section{Change of clinical manifestation according to diagnosis period}

When patients were compared according to period of diagnosis in intervals of 10 years, parenchymal NCC tended to decrease, although this was not statistically significant (Fig. 2). Among patients with parenchymal NCC, the proportion of viable and calcified parenchymal NCC did not change. Interest-

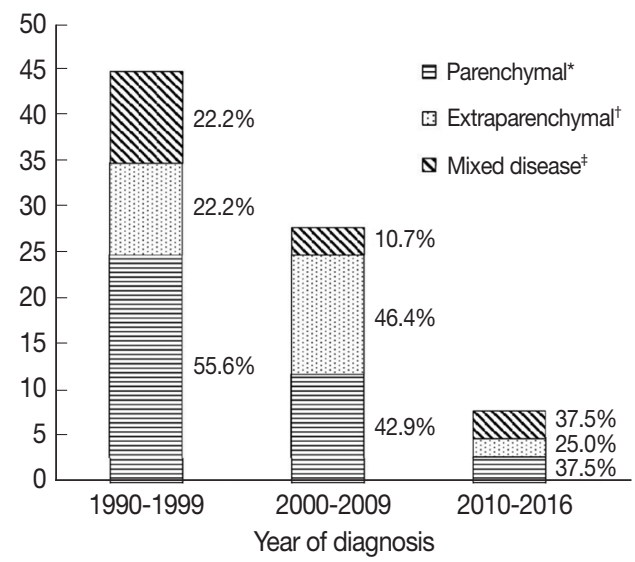

Fig. 2. Number of patients according to lesion location. *Parenchymal disease included cases with the nodules, cysts or calcifications located only in the parenchyma. 'Extraparenchymal disease included cases with cystic lesions in the ventricles or in the subarachnoid spaces. ${ }^{\ddagger}$ Mixed disease included cases with both parenchymal and extraparenchymal lesions. 
ingly, patients presenting with seizure significantly decreased over time ( $28.9 \%$ vs $14.3 \%$ vs $0 \%, P=0.033$ ), while gait disturbance increased ( $8.9 \%$ vs $17.9 \%$ vs $37.5 \%, P=0.037$ ).

\section{DISCUSSION}

In this retrospective review, we found that NCC cases are decreasing, and that mean age at diagnosis increased with time, although the data are limited to a single center. Nearly half of the patients had parenchymal NCC, while the other patients had either extraparenchymal NCC or mixed NCC. Patients with parenchymal NCC tended to present with seizure. By contrast, patients with extraparenchymal NCC more frequently presented with headaches. Most patients with extraparenchymal NCC had cysts in the subarachnoid space. Hydrocephalus and inflammatory changes such as high CSF protein and low glucose were more common in patients with extraparenchymal NCC. More patients with extraparenchymal NCC required a VP shunt placement relative to patients with parenchymal NCC.

The present study represents the decreasing NCC following the decrease of human taeniasis in Korea. Like other parasitic diseases, human taeniasis was common in Korea, with stool exams reporting an egg positive rate of approximately 1.0\% until the 1980s [7]. A series of national intestinal parasite surveillance studies identified that the prevalence decreased to lower than $0.1 \%$ in 1992, and no cases were found in 2004 $[8,9]$. In a recent national survey performed in 2012, the prevalence was also maintained below $0.1 \%$, consisting of 10 cases among the surveyed population of 23,956 (0.04\%) [10]. Since the 2012 survey, only sporadic cases of $T$. saginata infection have been reported, with no cases of T. solium infection [11]. Socio-economic changes have likely contributed to the decline of human taeniasis in the 1980s to 1990s. The introduction of praziquantel, economic development, changes in pig agriculture, use of commercial food, and improvement of the sewage system may have influenced the decline of human taeniasis collectively. The mean patient age in the present study is higher than that reported in previous studies [12]. The higher age at diagnosis suggests that most patients with newly presenting NCC were infected with T. solium eggs when the prevalence of human taeniasis was still high. More than $93.0 \%$ of patients in the present study were born prior to the 1960s.

The actual number of NCC cases is not well-characterized because it is not a notifiable disease. A few reports estimated the number of cysticercosis cases among pathology specimens without detailed case descriptions $[13,14]$. Although a decreasing trend of cysticercosis was suggested in a prior report [7], the study had limitations, including an ambiguous inclusion criteria. It is also difficult to estimate the incidence of NCC from our study, although it is suggestive of decreased NCC incidence. Because most NCC cases were diagnosed between the ages of 20 and 50 years [15-17], it appears plausible that new cases are becoming scarce. Conversely, our study found that NCC is still present, and should be considered as a differential diagnosis of patients presenting with seizure or headache. The youngest patient in our study was born in 1980, when taeniasis began to decrease. More than 25 years have passed, during which time the prevalence of taeniasis has been maintained below $0.1 \%$. However, our study suggests that NCC cases with a long incubation period still occur in Korea. Although the median incubation period of cysticercosis is around 3.5 years, incubation periods greater than 10 years have been reported [18].

The location of the parasite in the central nervous system was an important determinant of clinical characteristics in previous studies [12]. Our study also classified patients into parenchymal, extraparenchymal, and mixed NCC. Symptoms, CSF characteristics, proportion of hydrocephalus, and requirement for a VP shunt differed between parenchymal and extraparenchymal NCC. Patients with mixed NCC had similar clinical characteristics to patients with extraparenchymal NCC. These findings are consistent with previous studies [12].

In our study, $79.0 \%$ of patients were male. In male patients, the predominant subgroup was parenchymal NCC (54.7\%). However, female patients tend to develop extraparenchymal NCC (52.9\%). The reason for this sex-specific response is not clear. Previous studies found that women were more likely to have CSF pleocytosis and higher cell counts [12,19], which is associated with an inflammatory response. Immune-endocrine effects may contribute to increased inflammation in female patients $[12,19,20]$. However, CSF profiles suggestive of more inflammatory changes were not present in female patients in our study, so immune-endocrine effects are less likely to explain the association between extraparenchymal NCC and female sex in the present study.

Recent guidelines classified NCC into viable parenchymal NCC, calcified parenchymal NCC, single solitary granuloma, intraventricular NCC and subarachnoid NCC [5]. We also sought to apply this classification system in our patients. No 
patient had a single enhancing lesion. Forty-five percent of patients with parenchymal NCC had both viable and calcified lesions. Compared with calcified parenchymal NCC patients, those with viable parenchymal NCC tended to present with focal neurologic deficits, although this relationship was not statistically significant. Patients with calcified parenchymal NCC tended to develop seizure and clinical complications. The majority of patients with extraparenchymal NCC had subarachnoid lesions. Clinical complications tended to occur more commonly in the subarachnoid NCC group, although this trend also did not reach statistical significance.

Interestingly, in our study, we observed that clinical manifestation of NCC changed according to the period of diagnosis. Initially, we expected that calcified lesions would increase. However, the proportion of calcified parenchymal NCC did not change, while the proportion of patients presenting with seizure decreased. Contrastingly, the proportion of patients with extraparenchymal lesions increased, and patients were more likely to present with gait disturbance, which is suggestive of intracranial hypertension. Because subarachnoid NCC is more common in older patients [1,21], we suggest this finding implies that patients with longer incubation periods account for recent NCC cases, while new infections are increasingly rare. Notably, patients with spinal cord NCC experienced high rates of complications such as spinal cord compression (33.3\%) and vascular infarction (11.1\%).

The present study has several limitations. First, it only included patients with positive anti-cysticercus antibodies present in the CSF. Patients with positive serum antibodies but negative CSF antibodies were excluded from the study to increase the specificity. In our study, the anti-cysticercus antibody was measured by ELISA with the crude antigen from cysts of pig meat. This method is generally less specific than the enzyme-linked immunotransfer blot method. When using ELISA, performance was better for the CSF samples [22]. Serum ELISA results poorly correlate with clinical diagnosis [23]. Second, parasitological confirmation was insufficient in the patients with NCC. Pathological results were available only in $32.1 \%$ of patients and the result could not differentiate T. solium from other Taenia species. Third, this study is a single-centered retrospective study in South Korea. We cannot say that we have accurately apprehended the whole phenomenon of NCC in Korea from a single source of university hospital. For our findings to be generalizable to all of Korea, multicenter studies with a larger case series are required. Fourth, we could not determine in this study whether the NCC patients were from autochthonal taeniasis or from abroad. Due to the long incubation period of NCC, travel history was not informative.

In conclusion, our study suggests the prevalence of NCC is decreasing following the decline of human taeniasis in Korea. Subarachnoid extraparenchymal NCC with a longer incubation period has tended to predominate recently, suggesting that new cases are scarce. Since human taeniasis is considered to have almost disappeared from Korea, the number of NCC is also expected to decrease further. The clinical characteristics and outcomes of NCC were consistent with previous studies. Lesion location was an important determinant of clinical outcomes, which is also in line with previous studies. Because endemic infections have significantly decreased, risk factors and treatment outcomes of imported cases should be investigated in future studies.

\section{CONFLICT OF INTEREST}

The authors declare no conflict of interest related to this study.

\section{REFERENCE}

1. Garcia HH, Nash TE, Del Brutto OH. Clinical symptoms, diagnosis, and treatment of neurocysticercosis. Lancet Neurol 2014; 13: $1202-1215$.

2. Gripper LB, Welburn SC. Neurocysticercosis infection and disease-a review. Acta Trop 2017; 166: 218-224.

3. Baird RA, Wiebe S, Zunt JR, Halperin JJ, Gronseth G, Roos KL. Evidence-based guideline: treatment of parenchymal neurocysticercosis: report of the guideline development subcommittee of the American Academy of Neurology. Neurology 2013; 80: 1424-1429.

4. Choi MH, Yu JR, Hong ST. Who neglects neglected tropical diseases?-Korean perspective. J Korean Med Sci 2015; 30 (suppl): 122-130.

5. White AC, Coyle CM, Rajshekhar V, Singh G, Hauser WA, Mohanty A, Garcia HH, Nash TE. Diagnosis and treatment of neurocysticercosis: 2017 clinical practice guidelines by the infectious diseases society of America (IDSA) and the American society of tropical medicine and hygiene (ASTMH). Am J Trop Med Hyg 2018; 98: 945-966.

6. Kim SK, Wang KC, Paek SH, Hong KS, Cho BK. Outcomes of medical treatment of neurocysticercosis: a study of 65 cases in Cheju island, Korea. Surg Neurol 1999; 52: 563-569.

7. Chai JY. Human taeniasis in the Republic of Korea: hidden or gone? Korean J Parasitol 2013; 51: 9-17.

8. Korean Association of Health Promotion. Prevalence of Intesti- 
nal Parasitic Infections in Korea-the 7th report. Seoul, Korea. Korean Association of Health Promotion. 2004, pp 1-93.

9. Ministry of Health and Welfare, Korea Association of Health. Prevalence of Intestinal Parasitic Infections in Korea-the 6th report. Seoul, Korea. Ministry of Health and Welfare; Korea Association of Health. 1997.

10. Korea Centers for Disease Control and Prevention, National Institute of Health. The 8th National Surveys on the Prevalence of Intestinal Parasitic Infections. Seoul, Korea. Korean Association of Health Promotion. 2013, pp 1-287.

11. Cho J, Jung BK, Lim H, Kim MJ, Yooyen T, Lee D, Eom KS, Shin EH, Chai JY. Four cases of Taenia saginata infection with an analysis of COX1 gene. Korean J Parasitol 2014; 52: 79-83.

12. Marcin Sierra M, Arroyo M, Cadena Torres M, Ramirez Cruz N, Garcia Hernandez F, Taboada D, Galicia Martinez A, Govezensky T, Sciutto E, Toledo A, Fleury A. Extraparenchymal neurocysticercosis: demographic, clinicoradiological, and inflammatory features. PLoS Negl Trop Dis 2017; 11: e0005646.

13. Choi WH, Chu JP, Jiang M, Lee YS, Kim BS, Kim DG, Park YK. Analysis of parasitic diseases diagnosed by tissue biopsy specimens at KyungHee Medical Center (1984-2005) in Seoul, Korea. Korean J Parasitol 2010; 48: 85-88.

14. Kim J, Chung WS, Cho KH. Status of parasitic infection diagnosed by surgical biopsy in Kwangju and Chollanam-do. Korean J Parasitol 1994; 32: 93-100 (in Korean).

15. Croker C, Reporter R, Mascola L. Use of statewide hospital discharge data to evaluate the economic burden of neurocysticercosis in Los Angeles county (1991-2008). Am J Trop Med Hyg 2010; 83: 106-110.

16. Martinez HR, Rangel-Guerra R, Arredondo-Estrada JH, Marfil A,
Onofre J. Medical and surgical treatment in neurocysticercosis a magnetic resonance study of 161 cases. J Neurol Sci 1995; 130: 25-34.

17. Serpa JA, Graviss EA, Kass JS, White AC, Jr. Neurocysticercosis in Houston, Texas: an update. Medicine (Baltimore) 2011; 90: 8186.

18. Sorvillo F, Wilkins P, Shafir S, Eberhard M. Public health implications of cysticercosis acquired in the United States. Emerg Infect Dis 2011; 17: 1-6.

19. Fleury A, Dessein A, Preux PM, Dumas M, Tapia G, Larralde C, Sciutto E. Symptomatic human neurocysticercosis: age, sex and exposure factors relating with disease heterogeneity. J Neurol 2004; 251: 830-837.

20. Kelvin EA, Carpio A, Bagiella E, Leslie D, Leon P, Andrews H, Hauser WA. The association of host age and gender with inflammation around neurocysticercosis cysts. Ann Trop Med Parasitol 2009; 103: 487-499.

21. Fleury A, Escobar A, Fragoso G, Sciutto E, Larralde C. Clinical heterogeneity of human neurocysticercosis results from complex interactions among parasite, host and environmental factors. Trans R Soc Trop Med Hyg 2010; 104: 243-250.

22. Rodriguez S, Dorny P, Tsang VC, Pretell EJ, Brandt J, Lescano AG, Gonzalez AE, Gilman RH, Garcia HH. Detection of Taenia solium antigens and anti-T. solium antibodies in paired serum and cerebrospinal fluid samples from patients with intraparenchymal or extraparenchymal neurocysticercosis. J Infect Dis 2009; 199: 1345-1352.

23. Jin Y, Kim EM, Choi MH, Oh MD, Hong ST. Significance of serology by multi-antigen ELISA for tissue helminthiases in Korea. J Korean Med Sci 2017; 32: 1118-1123. 
